\title{
RADIOCARBON DATING OF SOILS: DATABASE CONTRIBUTION BY BONN AND HAMBURG
}

\section{H. W. SCHARPENSEEL, FRANZ PIETIG, HEINRICH SCHIFFMAN and PETER BECKER- HEIDMANN}

Institut für Bodenkunde, University of Hamburg, Allende-Platz 2, D-20146 Hamburg, Germany

ABSTRACT. We present a compilation of ${ }^{14} \mathrm{C}$ soil dates measured at the University of Hamburg through 1984 (HAM-1597).

\section{INTRODUCTION}

The inherent problems in determining the "age of a soil" were described by Scharpenseel and Becker-Heidmann (1992). The dating procedure applied in the production of ${ }^{14} \mathrm{C}$ dates of soil profiles and samples listed below was described by Scharpenseel, Pietig and Tamers (1968) and Scharpenseel and Pietig (1970). For reasons of brevity, we report only the oldest ${ }^{14} \mathrm{C}$ age of the data set indicated for the layers, thin layers or horizons of the ${ }^{14} \mathrm{C}$-dated soil profiles. We present an annotated bibliography as well as date lists of our work. We also include unpublished soil dates measured in our laboratory, and, following this contribution, Peter Becker-Heidmann reports continuing dates from HAM-1600 onward.

DATE LISTS IN RADIOCARBON: BONN I-VII AND HAMBURG I-IV*

\begin{tabular}{lll}
\hline Material dated & Lab code(s) & Age (yr BP) \\
\hline
\end{tabular}

University of Bonn, Natural Radiocarbon Measurements I (Scharpenseel, Pietig and Tamers 1968) Soil and Soil Profile Dates

\section{Germany}

Hapludoll, Söllingen A

Hapludoll, Söllingen B

$\begin{array}{lr}\text { B } 26-31 & \leq 4800 \pm 100 \\ \text { B 33-40 } & \leq 4060 \pm 80 \\ \text { B 98-104 } & \leq 5300 \pm 80 \\ \text { B 106-113 } & \leq 5550 \pm 80 \\ \text { B } 114-120 & \leq 3130 \pm 70 \\ \text { B 121-128 } & \leq 4000 \pm 80 \\ \text { B 3 } & 4000 \pm 80 \\ \text { B 4 } & 4170 \pm 80 \\ \text { B 22 } & 2560 \pm 60 \\ \text { B 96 } & 9130 \pm 100 \\ \text { B } 156 & \leq 1850 \pm 70 \\ \text { B } 161-165 & \leq 1390 \pm 70 \\ \text { B 9-13 } & \leq 1300 \pm 80 \\ \text { B 43-48 } & \leq 1220 \pm 80 \\ \text { B } 49-54 & \leq 1260 \pm 60 \\ \text { B } 129-135 & \leq 1190 \pm 70 \\ \text { B } 14 & \\ \text { B } 19 & 930 \pm 80 \\ \text { B 20, 21 } & 810 \pm 50\end{array}$

Hapludoll, Söllingen C

Hapludoll, Söllingen D

Haplaquoll, Hildesheim A

Haplaquoll, Hildesheim A

Fossil Chernozem below Hapludalf, Soest II

Fossil Chernozem below Hapludalf, Soest I

Fossil Chernozem in dark-brown steppe soil, Wallertheim

Buried Eutrochrept below Allerød trachyt blanket

Udoll, Ostholsteen A, Grossenbrode

Udoll, Ostholsteen B, Grossenbrode

Plaggept, Greven (Albachtenesch, Marktesch, etc.)

Plaggept, Greven, Albachtenesch, whole profile

Plaggept, Rheine

Plaggept, Lengerich (B $135,80 \mathrm{~cm}$ deep $3960 \pm 80$ )

Spodosol, Sennesand

Spodosol, Irrel

Spodosols, Darlaten A and B

B 20, 21

*From 1968-1974 our laboratory was located in Bonn, and our laboratory code designation, as reported in Radiocarbon, was BONN. In 1976, our laboratory numbers changed to HAM- to reflect our relocation to Hamburg. (Lab codes in this table are abbreviated to " $\mathrm{B}$ " and " $\mathrm{H}$ ".) This compilation of our work represents 27 years of soil dating from all over the world. 


\begin{tabular}{llc}
\hline Material dated & Lab code(s) & Age (yr BP) \\
\hline Spodosols, Scherpenseel A and B & B 90, 91 & $\leq 2960 \pm 70$ \\
Spodosol, Wilsede & B 41 & $1140 \pm 60$ \\
Spodosol, Obrehaverbeck & B 42 & $940 \pm 50$ \\
Spodosols, Flaesheim & B 15-17 & $\leq 2420 \pm 80$ \\
Hapludalf, Frimmersdorf & B 92-95 & $\leq 1880 \pm 80$ \\
Half bog soils, Fibrist, Kalkarer Moor I & B 82-85 & $\leq 7790 \pm 110$ \\
Half bog soils, Fibrist, Kalkarer Moor II & B 86-89 & $\leq 3160 \pm 50$ \\
\hline
\end{tabular}

University of Bonn, Natural Radiocarbon Measurements II (Scharpenseel, Pietig and Tamers 1969)

\section{Germany}

Rendolls

Tangelrendsina, Kramer (German Alps)

Moderrendsina Krottenkopf (German Alps)

Udalfs, argillic horizon probably former A horizon of Mollisol: Parabrown earth, Eltville

Parabrown earth, Inden

Brown earth, Haaren-Sintfeld

Parachernozem, Fellbach

Parachernozem, Fellbach, brickpit

Plaggepts:

Southeast of Rietberg

Brede near Rietberg

Hoffeld

Sinnesche Brede

Am Hohen Lande

Krax bei Neuenkirchen

Modern bomb-carbon samples BONN 172-200 and BONN 303-317

(bomb C curve on cereals, beets, winter rape, wine, 1956-1967)

$\begin{array}{lr}\text { B 318-322 } & \leq 4180 \pm 70 \\ \text { B 324 } & 600 \pm 50 \\ \text { B 326-331 } & \leq 4940 \pm 80 \\ \text { B 334-342 } & \leq 4170 \pm 70 \\ \text { B 355-358 } & \leq 1580 \pm 50 \\ \text { B 372-379 } & \leq 2730 \pm 70 \\ \text { B 380-384 } & \leq 4150 \pm 50 \\ & \\ \text { B 343-345 } & \leq 1200 \pm 70 \\ \text { B 348 } & 720 \pm 70 \\ \text { B 349-350 } & \leq 1130 \pm 70 \\ \text { B 351 } & 1540 \pm 60 \\ \text { B 352 } & 810 \pm 70 \\ \text { B 353 } & 900 \pm 60\end{array}$

University of Bonn, Natural Radiocarbon Measurements III (Scharpenseel and Pietig 1970)

Udolls, Vertisols, Fossil A-horizon of Paleosol-Mollisol in Argillic Horizon of Hapludalf

\section{Germany}

Parabrown earth Lantershofen

Ochtendung (below trachytic pumice)

Muddersheim, Thineland

Quarry "Schäferkalkwerke"

Buried soil organic matter (SOM), Eddersheim

Humus containing sand with charcoal (fireplace), Amalienhof

Buried humus, Heiligensee Forest, Berlin

Bone collagen in paleosol below trachytic tuff, Michelsberg

Bohemia, Czech Republic

Argiudoll, Kozojedy, Jicin District

Agiudoll, Smince, Uradec, Kralové District

Hapludoll, Brazdim, Prahoviphod, Tilery District

Vertisol (Smonitza), Prunevor, Choumtov District

Hapludoll, Chernozem, Zozelice, Königgrätz District

Aquoll, Zozelice II, Königgrätz District

Moravia, Czech Republic

Argiudoll, Brnicko, Olmütz District

Udoll, sandy loess, Moravia

Udoll, Chernozem, Bilorice

Vertic Udoll (Vertisol-like Chernozem), Tegel, Pole, Brünn

$\begin{array}{lr}\text { B 403-409 } & \leq 5530 \pm 90 \\ \text { B 411-416 } & \leq 10,580 \pm 100 \\ \text { B 417-421 } & \leq 3700 \pm 60 \\ \text { B 422-431 } & \leq 25,000 \pm 700 \\ \text { B 448 } & 8300 \pm 120 \\ \text { B 608a } & 2530 \pm 70 \\ \text { B 609 } & 760 \pm 60 \\ \text { B 763 } & 10,800 \pm 100\end{array}$

B $437-440$

B 441

B $442-444$

B 445-447

B 485-487

B $488-490$

$$
\begin{aligned}
& 4150 \pm 90 \\
& 4020 \pm 60 \\
\leq & 3430 \pm 65 \\
\leq & 6370 \pm 65 \\
\leq & 1460 \pm 110 \\
\leq & 1950 \pm 70
\end{aligned}
$$

B 491, 495

B $496-499$

B $500,600-603$

$\leq 4055 \pm 80$

$\leq 3610 \pm 75$

$\leq 2450 \pm 70$

$\leq 4070 \pm 70$ 


\begin{tabular}{|c|c|c|}
\hline Material dated & Lab code(s) & Age (yr BP) \\
\hline \multicolumn{3}{|l|}{ Russia } \\
\hline $\begin{array}{l}\text { Hapludoll, Vermudoll, Chernozem, Orel } \\
\text { Udoll, Chernozem, Charkov } \\
\text { Udoll, Chernozem, Zaparoskje } \\
\text { Udoll, Chestnut soil, Askania Nova }\end{array}$ & $\begin{array}{l}\text { B } 455-457 \\
\text { B } 460-462 \\
\text { B } 464-466 \\
\text { B } 468-470\end{array}$ & $\begin{array}{l}\leq 4720 \pm 60 \\
\leq 5920 \pm 140 \\
\leq 3270 \pm 80 \\
\leq 2710 \pm 80\end{array}$ \\
\hline \multicolumn{3}{|l|}{ Tunisia } \\
\hline $\begin{array}{l}\text { Vertisol, Beja (deepest humus layer) } \\
\text { Vertisol, Zouarine, Ebba Ksour }\end{array}$ & $\begin{array}{l}\text { B } 433 \\
\text { B } 434\end{array}$ & $\begin{array}{l}2920 \pm 40 \\
3680 \pm 65\end{array}$ \\
\hline \multicolumn{3}{|l|}{ Finland } \\
\hline $\begin{array}{l}\text { Sandy humus } 75 \mathrm{~cm} \text { deep, Kevo, North Finlandia } \\
\text { Spitzbergen }\end{array}$ & B 449 & $2350 \pm 70$ \\
\hline Fossil A horizon, $55 \mathrm{~cm}$ deep, Hohenstaufen Plateau, Barents I & B 432 & $3040 \pm 80$ \\
\hline \multicolumn{3}{|l|}{ Germany } \\
\hline $\begin{array}{l}\text { SOM fractions } \\
\text { Chernozem, Söllingen, total organic matter } \\
\text { Chernozem, Söllingen, humic acid extract only } \\
\text { Spodosol, Scherpenseel, brown humic acid fraction } \\
\text { Spodosol, Scherpenseel, gray humic acid fraction } \\
\text { Spodosol, Scherpenseel, rim of gravel pit } \\
\text { Spodosol, Scherpenseel, hymatomelanic acid fraction } \\
\text { Spodosol, Scherpenseel, brown humic acid fraction } \\
\text { Spodosol, Scherpenseel, gray humic acid fraction } \\
\text { Spodosol, Scherpenseel, humin fraction } \\
\text { Histosol, Kalkarer Moor, fulvic acid fraction } \\
\text { Histosol, Kalkarer Moor, hymatomelanic acid fraction } \\
\text { Histosol, Kalkarer Moor, brown humic acid fraction } \\
\text { Histosol, Kalkarer Moor, gray humic acid fraction } \\
\text { Histosol, Kalkarer Moor, humin fraction } \\
\text { Histosol, Kalkarer Moor, humus coal fraction } \\
\text { Aquoll, pseudogley-Chernozem, Adlum, fulvic acid fraction } \\
\text { Aquoll, pseudogley-Chernozem, hymatomelanic acid fraction } \\
\text { Aquoll, pseudogley-Chernozem, brown and gray humic acid fraction } \\
\text { Aquoll, pseudogley-Chernozem, humin fraction } \\
\text { Aquoll, pseudogley-Chernozem, humus coal fraction }\end{array}$ & $\begin{array}{l}\text { B } 6 \text { A } \\
\text { B } 6 \text { B } \\
\text { B } 138 \\
\text { B } 139 \\
\text { B } 366 \\
\text { B } 367 \\
\text { B } 368 \\
\text { B } 369 \\
\text { B } 370 \\
\text { B } 360 \\
\text { B } 361 \\
\text { B } 362 \\
\text { B } 363 \\
\text { B } 364 \\
\text { B } 365 \\
\text { B } 397 \\
\text { B } 398 \\
\text { B } 399 \\
\text { B } 401 \\
\text { B } 402 \\
\end{array}$ & $\begin{array}{l}2100 \pm 80 \\
2240 \pm 80 \\
2060 \pm 60 \\
1720 \pm 60 \\
2930 \pm 40 \\
1580 \pm 80 \\
2530 \pm 60 \\
2980 \pm 70 \\
2850 \pm 70 \\
4270 \pm 80 \\
4510 \pm 80 \\
5380 \pm 80 \\
5970 \pm 40 \\
3490 \pm 70 \\
4460 \pm 80 \\
1800 \pm 60 \\
1390 \pm 70 \\
4890 \pm 50 \\
2980 \pm 70 \\
2810 \pm 60\end{array}$ \\
\hline
\end{tabular}

University of Bonn, Natural Radiocarbon Measurements IV (Scharpenseel and Pietig 1971)

\section{Soil Profiles}

Hungary

Udalf, Chernozem, Erd, southeast Budapest

Udalf, Chernozem in sand-loess, Balatonföldvár, south bank of Lake Balaton

Udalf, Chernozem in fine sandy loess, Koszarhegy

Udalf, meadow soil, Boconad, east-northeast Budapest

Eutrochrept, Brown earth in loess, Kapoly

Hapludalf, Nagyrésce, southeast Budapest (below $112 \mathrm{~cm},{ }^{14} \mathrm{C}$ age jumps to $16,750 \pm 290$ )

Natrustalf, Hortobagy, southwest Debrecen, Rusta Plain

Histosol, bog soil, Nadasdladany, northeast of Lake Balaton

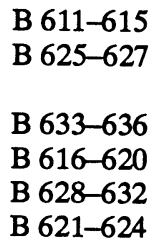

B $621-624$

B $648-651$

B $637-647$

B 458 $\leq 9680 \pm 100$

$\leq 4690 \pm 60$

$\leq 4575 \pm 60$

$\leq 5260 \pm 50$

$\leq 3990 \pm 70$

$\leq 2870 \pm 115$

$\leq 10,080 \pm 160$

$\leq 9300 \pm 340$

$12,470 \pm 360$ 


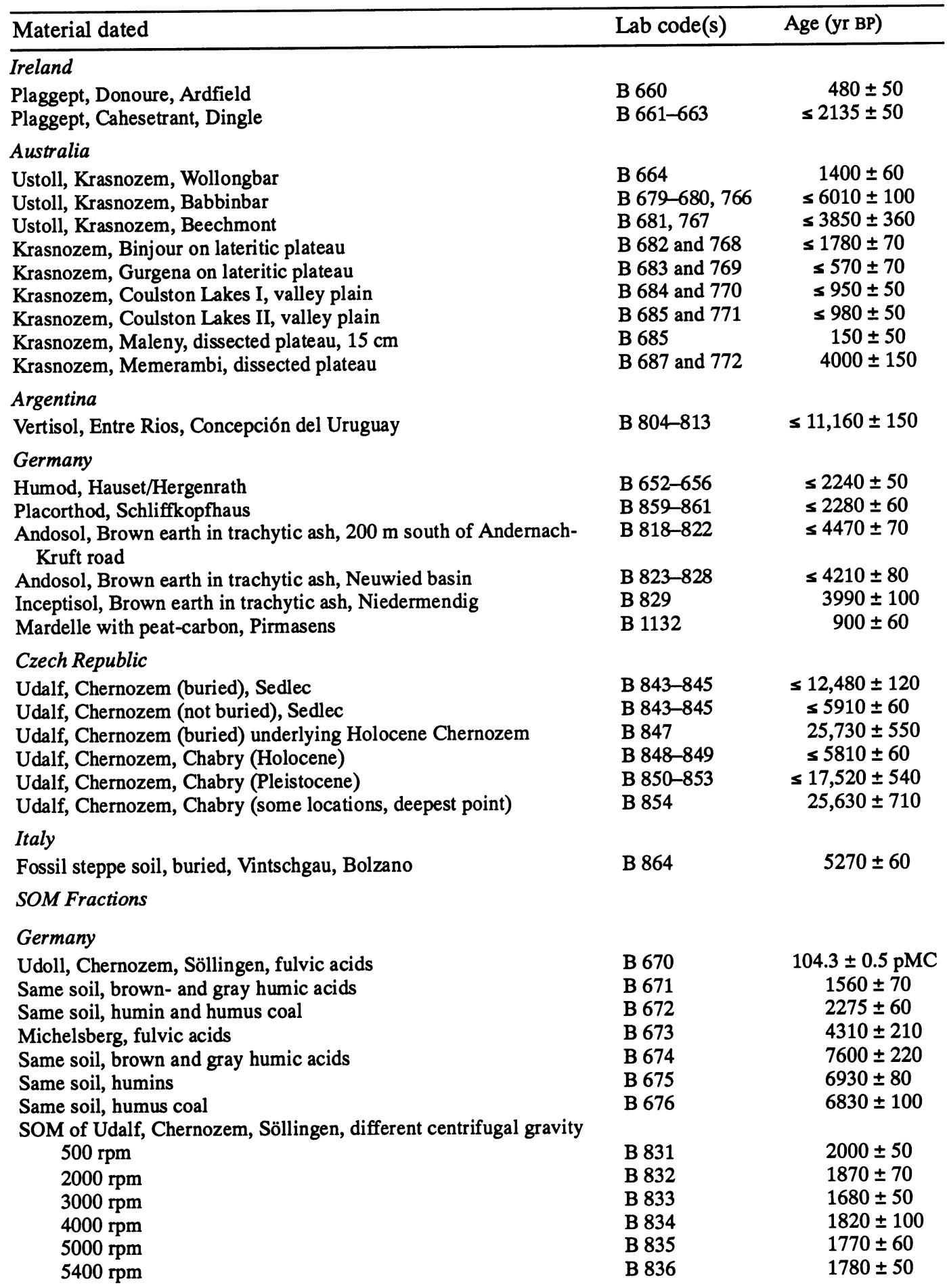




\begin{tabular}{llc}
\hline Material dated & Lab code(s) & Age (yr BP) \\
\hline $\begin{array}{l}\text { Hapludalf, Inden, fossil A horizon in } \mathrm{B}_{\mathrm{t}} \text { (argillic horizon), varying } \\
\text { texture fractions }\end{array}$ & & \\
$\quad>60 \mu \emptyset$ & B 1133 & $3170 \pm 80$ \\
$\quad 60-2 \mu$ & B 1134 & $3450 \pm 80$ \\
$2-1 \mu$ & B 1135 & $3280 \pm 80$ \\
$1-0.5 \mu$ & B 1136 & $2790 \pm 770$ \\
$\quad 0.5-0.25 \mu$ & B 1137 & $2500 \pm 70$ \\
Subhydrous Soils, Gyttja & & \\
Germany & & \\
Schalkenmeerer Maar, 0-230 cm & & \\
Lake of Selent, gyttja, 0-560 cm & B 781-802 & $\leq 4600 \pm 70$ \\
\hline
\end{tabular}

\section{University of Bonn, Natural Radiocarbon Measurements V (Scharpenseel and Pietig 1973a)}

Soil Profiles

Israel

Xeralf, Hamra, below dune cover, Tel Aviv-Haifa highway, corner of Richlon Street

Xeralf, same location, foot of slope, no continuous dune cover

Xeralf, same location, emerging into recent soil, no dune cover

Xeralf, Hamra, in dune material with lime concretions (Curcar), Wingate Institute of Athletics, near Tel Aviv-Haifa highway

Xeralf, calcinated root in Hamra, street to Ecron

Curcar-Hamra sequence, Rehovot, corner of Main Street and Batia Markov

Aqualf, Nazas, Jashresh

Soil Assocations on Limestone

Xeroll on soft limestone, Mitzpe Mesua

Xerochrept, calcareous brown earth on harder limestone, near Mitzpe Mesua

Rhodustalf-Terra Rossa on hard limestone, Mattah

Xeralft with recalcification (Husmas soil), Agricultural School, Kanot

Xeroll, Burozem, overlying Xeralf with recalcification (Husmas),

Kibbutz Ruchama

Xeroll

Underlying recalcified Xeralf

Fossil clay below recalcified Xeralf

Dark brown soil in calcareous dune sand, chesnut-like soil, Mafkiim, south of Ashkalon

Palexeroll near Shuval, road from Beer Shewa to Tel Aviv

Paleorthid in loess, Eshel Hanassi, neer Beer Sheva

Xerert, west Plain of Barkai, road from Afula to Hedra

Xerert, Valley of Jesrael

Xerert, Valley of Jesrael, drainage ditch

Xerert, El Hamma

Xerert, near Kefar Manachem Kibbutz

Xerert along Syrian Quarantine Station and Jordan flow into Lake Genezareth

Bulgaria

Vertic Albaqualf, Glavatsi

Udic Haplustoll, leached Chernozem, near Gorni Dubnik
B 688-691

B 692-695

B 696-699

B 701-706

B 709

B 711

B 712, 713

$\leq 14,740 \pm 200$

$\leq 10,470 \pm 130$

$\leq 11,860 \pm 150$

$\leq 17,920 \pm 180$

$16,930 \pm 240$

$14,920 \pm 230$

$\leq 2960 \pm 220$

B 742,743

B 744

$\leq 1500 \pm 50$

$2040 \pm 60$

B 745

B 748, 749

$2420 \pm 70$

$\leq 5050 \pm 160$

B 751, 752

B 753-755

B 757

B 750

B 760-762

B 758-759

B 715-718

B 719-723

B 724-728

B 729-734

B 735-741

B 773-776

$\leq 9000 \pm 200$

$\leq 13,400 \pm 190$

$19,920 \pm 340$

$4760 \pm 80$

$\leq 15,470 \pm 230$

$\leq 4020 \pm 220$

$\leq 1850 \pm 70$

$\leq 2760 \pm 80$

$\leq 7440 \pm 80$

$\leq 19,430 \pm 350$

$\leq 16,100 \pm 270$

$\leq 2670 \pm 100$

B 1071-1074 $\leq 8050 \pm 80$

B 1075-1079 $\leq 11,100 \pm 90$ 


\begin{tabular}{|c|c|c|}
\hline Material dated & Lab code(s) & Age (yr BP) \\
\hline $\begin{array}{l}\text { Typic Caciustoll, calcareous Chernozem, northwest Pleven } \\
\text { Paleustalf, Gray Forest soil, } 12 \mathrm{~km} \text { south of Pleven } \\
\text { Udic Haplustalf, Gray Forest soil, Kozlevo-Shoumen } \\
\text { Udertic Paleustalf, degraded Cinnamon Forest soil, near Bourgas } \\
\text { Vertic Albaqualf, Cinnamonic, podzolized Planosol, Badeshte, Thra- } \\
\text { cian plain } \\
\text { Pellustert, Smonitsa-Vertisol, Sredets, Thracian Plain } \\
\text { Chromic Luvisol, Cinnamonic Forest soil, Koren }\end{array}$ & $\begin{array}{l}\text { B } 1080-1085 \\
\text { B } 1086-1092 \\
\text { B } 1093-1097 \\
\text { B } 1098-1104 \\
\text { B } 1105-1108 \\
\text { B } 1108-1114 \\
\text { B } 1115-1119\end{array}$ & $\begin{aligned} & \leq 5760 \pm 90 \\
\leq & 18,920 \pm 340 \\
\leq & 3370 \pm 100 \\
\leq & 14,150 \pm 240 \\
\leq & 9850 \pm 240 \\
\leq & 16,140 \pm 460 \\
& \leq 8480 \pm 140\end{aligned}$ \\
\hline $\begin{array}{l}\text { Sardinia } \\
\text { Xerert (aquic), Plane de Cuga, Ittiri, southwest Sassari } \\
\text { Chromoxerert, river terrace, Rio Mannu di S. Vero } \\
\text { Pelloxerert, Arziadas, Tuvoi } \\
\text { Chromoxerert, Monastir, } 20 \mathrm{~km} \text { north of Cagliari } \\
\text { Pellustert, Nurallo }\end{array}$ & $\begin{array}{l}\text { B } 1154-1157 \\
\text { B } 1141-1164 \\
\text { B } 1167-1174 \\
\text { B } 1175-1178 \\
\text { B } 1180-1187\end{array}$ & $\begin{array}{r}\leq 570 \pm 50 \\
\leq 3870 \pm 130 \\
5430 \pm 100 \\
\leq 2270 \pm 70 \\
\leq 3220 \pm 80\end{array}$ \\
\hline $\begin{array}{l}\text { Sicily } \\
\text { Chromoxerert, Scalilli near Corleone } \\
\text { Pelloxerert, Plana di Scala, Corleone } \\
\text { Pelloxerert, Aziena Sporacia, Farm, Università di Palermo } \\
\text { Pelloxerert, Aziena Sporacia, Farm, Università di Palermo }\end{array}$ & $\begin{array}{l}\text { B } 1326-1331 \\
\text { B } 1332-1338 \\
\text { B } 1339-1351 \\
\text { B } 1352-1363\end{array}$ & $\begin{aligned} & \leq 3030 \pm 90 \\
& \leq 3670 \pm 100 \\
& \leq 5470 \pm 120 \\
\leq & 15,160 \pm 370\end{aligned}$ \\
\hline $\begin{array}{l}\text { Romania } \\
\text { Humic horizon underlying Danube alluvium, near Bucharest }\end{array}$ & В 1379-1385 & $\leq 8070 \pm 130$ \\
\hline $\begin{array}{l}\text { Germany } \\
\text { Aqualfic Fragiorthod, Amelsbüren } \\
\quad \text { (below } 160 \mathrm{~cm}, 15,170 \pm 230 \text { ) } \\
\text { Placorthod, Grindenschwarzwald, Gemsbach }\end{array}$ & $\begin{array}{l}\text { В } 1363-1369 \\
\text { В } 1371-1377\end{array}$ & $\begin{array}{l}\leq 1980 \pm 80 \\
\leq 2550 \pm 70\end{array}$ \\
\hline
\end{tabular}

University of Bonn, Natural Radiocarbon Measurements VI (Scharpenseel and Pietig 1973b)

Soil Profiles

Spain

Xerert, La Rinconada (Seville), Casas vacas

B 1388-1392

B $1393-1397$

Xerert, Carmona, km 10.5 Carmona-Arahal Street

B 1398-1406

Xerert, El Arahal, Estrella Farm, $40 \mathrm{~km}$ east of Seville

B 1407-1413

Xerert, Carmona, La Motilla Chica Farm

B 1417-1423

Portugal

Xerert, Black Barros, Beja

Vertic Xerochrept, Safara-Camauros Farm

B 1425-1432

Xerert, Safara field

Red Xerert, Salvada-Beja

Roman grain silo in Xerert, Beja-Serpa

Dark red Xerert, Montes Velhos Aljustrel

B $1433-1437$

B $1438-1442$

B $1443-1446$

B 1447

B $1452-1457$

Red-brown Xerert, Terra Grande de Lisboa, Tapaiao da Ajuda, uni-

B 1458-1463

$\leq 6470 \pm 130$

$\leq 6650 \pm 120$

$\leq 8850 \pm 130$

$\leq 3480 \pm 100$

$\leq 3440 \pm 290$ versity campus near Lisbon

Australia

Ustert, Lillimur, Kaniva District, Victoria, Gilgai mound

Ustert, Lillimur, Kaniva District, Victoria, Gilgai depression

B $1466-1485$

B $1486-1507$

Ustert, Miram, Kaniva District, Victoria, Gilgai mound

B $1508-1527$

B 1528-1548

$\begin{aligned} \leq & 3070 \pm 140 \\ \leq & 2430 \pm 70 \\ \leq & 2230 \pm 190 \\ \leq & 1380 \pm 70 \\ & 5150 \pm 100 \\ \leq & 2240 \pm 80 \\ \leq & 3170 \pm 80\end{aligned}$

Ustert, Miram, Kaniva District, Victoria, Gilgai depression

$\leq 8450 \pm 260$ 


Lab code(s) Age (yr BP)

Material dated
Germany
Fossil A horizon, Eberspoint, $10 \mathrm{~km}$ west of Freising
Fossil A horizon, terrace brown earth, $5 \mathrm{~km}$ south of Siegenburg,
Abens Valley, north Bavaria
Charcoal from different fireplaces
Hapludalf in flood loam, lower terrace of the Rhine River
Fossil A horizon below Hapludalf, same location
Bituminous coating on gravel in younger Isar terrace, Ascholding,
Wolfratshausen Basin
Umbrept (Plaggept?), Würmian basal moraine of Isar foreland gla-
cier, Unterbuchen, Bavaria
Root in Maintag gravel pit, Upper Franconia
Fossil A horizon in Maintag gravel pit, Upper Franconia
Root, vertical in sediment, Maintag gravel pit
Fossil A horizon, covered by Pleistocene terrace material, Kärlich,
Rhineland
Humus in silty A horizon, below tuffaceous material, north slope of
Bausenberg, Lengsdorf, Rhineland
Fossil A horizon, gravelly gley, Gammelsbach valley, North Eber-
bach, Odenwald
Soil Fractions
Fossil A horizon, underlying Allerød trachytic tuff, charcoal only
Same location, particle size fraction $>0.2$ mm
Same location, particle size fraction $63-2 \mu$
SOM from continuous extraction, successive fractions (1st extrac-
tion: $0.1 \mathrm{~N} \mathrm{H}_{2} \mathrm{SO}_{4}$; 2 nd extraction: $0.15 \mathrm{M} \mathrm{Na}_{4} \mathrm{P}_{2} \mathrm{O}_{7}$ )
Udalf Aseler Holz:

Spodosol (Humod) gravel pit Weber, Scherpenseel (Dutch border):

Humod, Scherpenseel, Weber gravel pit

Subhydrous Soils

Gyttja, bottom of Schalkenmehrer Maar, Eifel I

Gyttja, bottom of Schalkenmehrer Maar, Eifel II

Gyttja, bottom of Schalkenmehrer Maar, Eifel IV

Gyttja, bottom of Schalkenmehrer Maar, Eifel V

Gyttja, bottom of Lake of Selent, Holsteen I

$2 \mathrm{~m}$ below end of case lot (sampling instrument)

Gyttja, bottom of Lake of Selent, Holsteen III

Gyttja, bottom of Lake of Selent, Holsteen IV

On-shore profile, Lake of Selent, Holsteen IV (opposite Profile IV)

Gyttja, bottom of Lake of Selent, Holsteen V

Gyttja, bottom of Lake of Selent, Holsteen VI

at $340 \mathrm{~cm}$ depth

B 1464
B 1648
B 1648,1649
B 1652
B 1653
B 1657

B $1669-1672$

B 1700

B 1801

B 1802

B 1659

B 1699

B 1815

B 1681

B 1681

B 1684

B 1809
B 1810
B 1811

B 1688

B 1689

B 1691

B 1692

B 1693

B 1697

$6160 \pm 90$

$2270 \pm 70$

$\leq 1460 \pm 70$

$5080 \pm 110$

$8230 \pm 470$

$28,320 \pm 470$

$\leq 3800 \pm 80$

$1810 \pm 70$

$7980 \pm 110$

$4360 \pm 90$

$30,450 \pm 1270$

$22,360 \pm 510$

$1350 \pm 110$

$11,550 \pm 160$

$7570 \pm 190$

$10,950 \pm 150$

B 994-1005

B 1007-1025

B 1026-1045

B 1046-1064

B 869-880,

1122-1125

B 911-932,

963-964

B 933-959

B 1127-1131

B 967-976

B 977-993
$1030 \pm 100$

$4130 \pm 270$

$4970 \pm 80$

$1400 \pm 140$

$1160 \pm 70$

$1460 \pm 80$

$1350 \pm 110$

$1510 \pm 130$

$1290 \pm 70$

$\leq 12,130 \pm 140$

$\leq 12,130 \pm 140$

$\leq 3840 \pm 80$

$\leq 1670 \pm 100$

$10,170 \pm 140$

$\leq 24,830 \pm 970$

$\leq 17,390 \pm 460$

$\leq 2670 \pm 70$

$\leq 14,180 \pm 670$

$\leq 10,080 \pm 520$

$30,930 \pm 1150$ $\leq 12,160 \pm 130$ 


\begin{tabular}{lll}
\hline Material dated & Lab code(s) & Age (yr BP) \\
\hline University of Bonn, Natural Radiocarbon Measurements VII (Scharpenseel and Pietig 1974) \\
Germany & & \\
Subhydrous Soils & & \\
Gyttja, bottom of Lake Laach, Eifel I & B $1572-1590$ & $\leq 23,010 \pm 460$ \\
Gyttja, bottom of Lake Laach, Eifel II & B $1591-1607$ & $\leq 20,500 \pm 380$ \\
Gyttja, bottom of Lake Laach, Eifel III & B $1609-1622$ & $\leq 22,070 \pm 440$ \\
Gyttja, bottom of Lake Laach, Eifel IV & B $1623-1636$ & $\leq 10,700 \pm 140$ \\
Gyttja, bottom of Lake Laach, Eifel V & B $1637-1643$ & $\leq 20,130 \pm 300$ \\
Gyttja, bottom of Meerfelder Maar, Eifel I & B $1818-1832$ & $\leq 6800 \pm 100$ \\
Gyttja, bottom of Meerfelder Maar, Eifel II & B $1838-1838$ & $\leq 9140 \pm 120$ \\
Gyttja, bottom of Meerfelder Maar, Eifel III & B $1839-1849$ & $\leq 7380 \pm 90$ \\
Gyttja, bottom of Meerfelder Maar, Eifel IV & B $1850-1859$ & $\leq 5550 \pm 90$ \\
Gyttja, bottom of Meerfelder Maar, Eifel V & B $1860-1867$ & $\leq 3520 \pm 80$ \\
Gyttja, bottom of Meerfelder Maar, Eifel VI & B $1868-1876$ & $\leq 4050 \pm 80$ \\
Gyttja, bottom of Pulvermaar, Eifel I & B $1877-1891$ & $\leq 8040 \pm 120$ \\
Gyttja, bottom of Pulvermaar, Eifel II & B $1892-1903$ & $\leq 6720 \pm 110$ \\
Gyttja, bottom of Pulvermaar, Eifel III & B $1904-1916$ & $\leq 6420 \pm 180$ \\
Gyttja, bottom of Pulvermaar, Eifel IV & B $1917-1930$ & $\leq 4980 \pm 80$ \\
Gyttja, bottom of Pulvermaar, Eifel VI & B $1931-1946$ & $\leq 5170 \pm 180$
\end{tabular}

Hamburg University, Radiocarbon Dates I (Scharpenseel, Pietig and Schiffmann 1976)

Germany

Soil samples and soil profile samples

Argillic horizon of Hapludalf, Friesdorf near Bonn

South side of same pit, Friesdorf

Aquept, alluvial loess, Isar terrace, Landshut/Ergolding $\quad$ H 4-7

Udoll high terrace of Isar, Landshut/Ergolding $\quad$ H 8-10

Hapludalf, high terrace of Isar, Lanshut/Ergolding $\quad$ H 11-13

Koislhof, lower to upper nether terrace, Isar River $\quad$ H 14-25

North Bavaria, north of Danube, humic colluvium on old Riss mo- $\quad$ H 26-31 raine north of Landsberg

Mollisol in steep bank of Lech River, Kaufering

Fossil organic matter in tuffaceous limestone, Neuenried, near sources of Mindel River

Fossil argillic horizon between terrace gravel and loess loam, Fellheim brick factory, Iller River

Eutochrept, Hohentrüdigen on Dogger (Jurassic)

Vertisol on Tertiary deposits in the Ries, Maihingen

Aquic Vertisol on younger Ries lake deposits, Pfäfflingen

Aquic Vertisol, Ries lake deposits Wechingen

Vertic Brown earth (Inceptisol), Ries lake deposits, Munningen

Folist, Nether Moor, Koislhof, near Landshut

Udalf Smonica/Chernozem, Lötzweiler, Rheinhessen

Mollisol/Hapludalf, Dorla I/2, Fritzlar-Gudensberg

Mollisol/Hapludalf, Lohne I, north of village of Lohne

Mollisol/Hapludalf, Wehren I/2, Wehren-Kirchberg

Mollisol/Hapludalf, Wehren I/3, Wehren-Kirchberg

Mollisol Heuchelheim, overlying degraded Chernozem

H 32-36

H 37-43

H 44-46

$5010 \pm 280$

$3620 \pm 70$

$\leq 10,880 \pm 140$

$\leq 3190 \pm 80$

$\leq 3320 \pm 70$

$\leq 10,680 \pm 140$

$\leq 4340 \pm 70$

$\leq 4410 \pm 80$

$\leq 4580 \pm 90$

Colluvium overlying degraded Chernozem, Bleichen II

H 47-49

$\leq 4340 \pm 130$

Loess on solifluction debris, south of Höingen, Vogelsberg

H 50-55

H 56-60

H 61, 62

H 63, 64

H 64

H 66, 67

H 68,69

$\mathrm{H} 70$

H 71

H 72

H 73

$\mathrm{H} 74$

H 75

H 76

Humus in solifluction debris, 300 m east of Taufstein, Vogelsberg

H 77, 78 


Material dated
High flood loam, Main River, Kelsterbach-Lerchenberg, Kiesgrube
Schmidt
Fossil epipedon, on Allerød trachyte, Buchslag, plain south of Main
River
Colluvial loam, Hochheim, Hattersheim, valley sediments
Colluvium, Waldeck (Freienhagen, Ippinghausen)
Spodosol, "Raseneisengley”, Black Forest, Altglashütten
Spodosol, Black Forest, between Breitnau and Hollertal
Charcoal and fossil Brown earth, Trescher, Black Forest
Low moor peat, charcoal, paleosol on Riss boulder marl, Altenerding,
Bavaria
Cliff wall at Baltic Sea coastline, Heiligenhafen
Fossil Chernozem (Udalf), outskirts of Homberg, Kassel County
Fossil Chernozem (Udalf), Worms I, near town of Worms
Bones, wood, sediments believed from Roman times, Xanten
Humic soils near Dutch border, organic matter from plaggen or deep
ploughing, total samples and $6 \mathrm{~N}$ HCl hydrolysis residue
Walbecker Heide
Maasaue near Velden
Haus Beerenbruck
Schandelah
Walbeck
Subhydrous soil samples, Baldeney lake, Essen-Werden, south shore
Clay pit Kaerlich, wood sample underlying Allerød and Holocene
soil

Age (yr BP)
$\leq 7900 \pm 100$

Wales

Placorthods, Hiraethog, Denbighmors, Denbigshire

Peny Gwrydd Pass, Snowdonia National Park, Caernarvonshire

Low moor (Histosol), Neumarkt, Am Wallersee, Salzburg

Former USSR

Predkaokadzye Chernozem (Ustoll), south Rostov

Chernozem, lower terrace of Don River, from krotovinas

Meadow Chernozem in Azow system

Dark Gray Forest soil, Zhiguli

Pelehydromorphic Chernozem, Zhiguli, krotovina

Chernozem Privolzhye Upland, Karlinsky Far, Ulyanovsk

Chernozem near Solod Meadow, Ulyanovsk Agricultural Institute campus

Gray Forest soil, Vysokogorsky, Tatar SSR

Chernozem, Tour I, 10th International Congress on Soil Science, Moscow

\section{Tunisia}

Paleosol in pasture, Ain Oktor, Korbous

Fossil horizons, Ferme Korba, pasture experiment

Fossil horizons near Ferme Korba, pasture experiment

Fossil horizons, Ferme Ennasser near Bir Bou Rekba

Fossil horizon near Enfida, $\mathrm{km}$ 52, road to Kairouan

Fossil horizon, Medjerdah alluvium near Ghardimaou bridge

Fossil horizons in Medjerdah alluvium, near Bou Salem

Fossil horizons, Medjerdah River, Bou Huertma River

Pale-Xerochrept, Sol Brun à Croûte (crust faintly developed), Medjerdah Valley, hill site

Lab code(s)
H 79,80
H 81
H $82-93$
H 94,95
H $96-101$
H $109-112$
H $113-117$
H $118-122$

H $123-127$
H 128
H 129
H $130-133$

H 134,135
H 136,137
H 138,139
H 140,141
H 142,143
H $183-188$
H 189

H $103-108$

H 105-106

H 144-145

$$
10,000 \pm 210
$$

$\leq 6970 \pm 100$

$\leq 820 \pm 110$

$\leq 3120 \pm 90$

$\leq 1120 \pm 60$

$\leq 1040 \pm 50$

$\leq 30,340 \pm 1130$

$$
\begin{array}{r}
\leq 4020 \pm 90 \\
5650 \pm 80 \\
3530 \pm 70 \\
\leq 3150 \pm 70
\end{array}
$$

$$
\begin{aligned}
& \leq 9580 \pm 100 \\
& \leq 2070 \pm 90 \\
& \leq 1860 \pm 90 \\
& \leq 1440 \pm 60 \\
& \leq 1880 \pm 130 \\
& \leq 12,160 \pm 270 \\
& 29,600 \pm 1150
\end{aligned}
$$

H 146

H 147

H 148

H 149

H 150-151

H 152

$\leq 5810 \pm 150$

$\leq 1770 \pm 80$

H 153

H 154

H 155-156

$\leq 3770 \pm 70$

H 157

H 158-160

H 161-162

H 163-164

H 165

H 166-167

H 168-171

H 172-174

H 175-182

$$
\begin{aligned}
11,330 & \pm 980 \\
5250 & \pm 150 \\
6510 & \pm 260 \\
5490 & \pm 280 \\
\leq 4750 & \pm 80 \\
5390 & \pm 120 \\
5550 & \pm 140 \\
& \\
7890 & \pm 680 \\
\leq 4540 & \pm 80
\end{aligned}
$$

$$
\begin{aligned}
& 2250 \pm 60 \\
\leq & 2300 \pm 100 \\
\leq & 2470 \pm 70 \\
\leq & 4230 \pm 60 \\
& 4510 \pm 80 \\
\leq & 8000 \pm 180 \\
\leq & 7800 \pm 160 \\
\leq & 2400 \pm 120 \\
\leq & 27,160 \pm 1090
\end{aligned}
$$




\begin{tabular}{|c|c|c|}
\hline Material dated & Lab code(s) & Age (yr BP) \\
\hline \multicolumn{3}{|c|}{ Hamburg University, Radiocarbon Dates II (Scharpenseel and Schiffmann 1977) } \\
\hline \multicolumn{3}{|l|}{ Argentina } \\
\hline $\begin{array}{l}\text { Argiudoll, Brunizem, Rafaela } \\
\text { Argiudoll, Brunizem, Esperanza } \\
\text { Argiudoll, slightly planosolic Brunizem, Angel Gallardo } \\
\text { Arguidoll, Profile B9, Villa Concepción del Tío }\end{array}$ & $\begin{array}{l}\text { H } 231-237 \\
\text { H } 238-244 \\
\text { H } 246-252 \\
\text { H } 253-257\end{array}$ & $\begin{array}{r}\leq 480 \pm 80 \\
\leq 2030 \pm 80 \\
\leq 2780 \pm 80 \\
\leq 1870 \pm 90\end{array}$ \\
\hline \multicolumn{3}{|l|}{ Tunisia } \\
\hline Paleosol in Bou Huertma alluvium & H 258-259 & $\leq 4930 \pm 80$ \\
\hline \multicolumn{3}{|l|}{ Germany } \\
\hline $\begin{array}{l}\text { Mud in old river bed of Ems River, on top of low moor, Rietberg } \\
\text { Ochrept, Holzkirchen, near Munich-Salzburg highway } \\
\text { Humic acid samples of Lower Saxonian soils } \\
\text { SOM fractions, Spodosol, Scherpenseel, near Dutch border } \\
\text { SOM fractions, Udoll, Aseler Wald, near Hildesheim } \\
\text { SOM fraction, paleosol of loess, below trachytic tuff of Allerød vol- } \\
\text { canism }\end{array}$ & $\begin{array}{l}\text { H } 280-281 \\
\text { H } 631-634 \\
\text { H } 260-279 \\
\text { H } 282-285 \\
\text { H } 286-297 \\
\text { H } 298-310\end{array}$ & $\begin{aligned} & \leq 6680 \pm 90 \\
& \leq 2510 \pm 50 \\
\leq & 19,800 \pm 710 \\
& \leq 5410 \pm 90 \\
& \leq 3160 \pm 70 \\
\leq & 11,360 \pm 150\end{aligned}$ \\
\hline $\begin{array}{l}\text { SOM fractions, vertic, clayey Histosol, Koislhof, lower terrace of Isar } \\
\text { River }\end{array}$ & H $311-328$ & $\leq 13,140 \pm 200$ \\
\hline $\begin{array}{l}\text { SOM fractions after repeated } 6 \mathrm{HCl} \text { hydrolysis, Udoll from Asel clay } \\
\text { pit near Hildesheim }\end{array}$ & H 623-630, 801 & $\leq 3260 \pm 100$ \\
\hline $\begin{array}{l}\text { SOM fractions after repeated } 6 \mathrm{HCl} \text { hydrolysis, histic Udoll, Ergold- } \\
\text { ing near Landshut }\end{array}$ & H 762-788, 800 & $\leq 6110 \pm 90$ \\
\hline Sea level-coast line study based on peat dating, North Sea shore & H 765-776 & $\leq 6130 \pm 240$ \\
\hline $\begin{array}{l}\text { Humus in Elbe River alluvium, Billwerder-Allermöhe, south Ham- } \\
\text { burg, measurement of deposition date }\end{array}$ & H 791-799 & $\leq 3370 \pm 100$ \\
\hline
\end{tabular}

Hamburg University, Radiocarbon Dates III (Scharpenseel, Schiffmann and Hintze 1984)

Germany

Histic Hapludoll, $5 \mathrm{~km}$ south of Söllingen, 0-90 cm, at 5-cm intervals Carbonate-free soil

$6 \mathrm{~N} \mathrm{HCl}$ hydrolysis residue

$6 \mathrm{~N} \mathrm{HCl}$ hydrolyzate

Acid from carbonate destruction

Typic Hapludoll, Söllingen, near old windmill, 0-75 cm, at $5-\mathrm{cm}$ intervals, carbonate-free soil

$6 \mathrm{~N} \mathrm{HCl}$ hydrolysis residue

$6 \mathrm{~N} \mathrm{HCl}$ hydrolyzate

Acid from carbonate destruction

Eutochrept, near Hohentrüdingen, Jurassic Dogger, near Nördlinger Ries crater, taken in 5-cm intervals, 5 to $105 \mathrm{~cm}$ depth

Haplaquept on Isar River terrace, near Landshut, Ergolding, Bavaria, taken in 5-cm intervals, 0 to $90 \mathrm{~cm}$ depth

Humic matter, coastline levee along Baltic coast, near Heiligenhafen

Elbe River marsh series, Allermöhe, Vier and Marschlande, south of Hamburg

(H 835, paleosol, $7420 \pm 110$ )

$\begin{array}{cc}\text { B 2255-2272 } & \leq 6210 \pm 90 \\ \text { B 2401-433 } & \leq 6370 \pm 80 \\ \text { B 2400-2428 } & \leq 3240 \pm 70 \\ \text { B 2438-2450 } & \leq 3570 \pm 70 \\ \text { B 2275-2289 } & \leq 2450 \pm 60 \\ & \\ \text { B 2476, 2478, } & \leq 3260 \pm 60 \\ 2484-2492, & \\ 2500,2502, & \\ 2504 & \\ \text { B 75, 2477, } & \leq 40 \pm 60 \\ 2483-2491, & \\ 2499,2501, & \\ 2503 & \leq 240 \pm 90 \\ \text { B 2459-2469 } & \\ \text { H 635-654 } & \leq 16,776 \pm 280 \\ \text { H 655-672 } & \leq 5560 \pm 80 \\ \text { B 2367-2385 } & \leq 6240 \pm 110 \\ \text { H 826-841 } & \leq 3970 \pm 80\end{array}$




\begin{tabular}{lcc}
\hline Material dated & Lab code(s) & Age (yr BP) \\
\hline $\begin{array}{l}\text { Peaty material, underlying valley of Elbe River, sampled in three } \\
\quad \text { cross-sections, east and west of Hamburg }\end{array}$ & H 1393-1406 & $\leq 8140 \pm 100$ \\
$\begin{array}{l}\text { Australia (see also B 664 to 772, Scharpenseel and Pietig (1973b: 258-263)) } \\
\text { Vertisol (Chromustert), Chinchilla }\end{array}$ & H 674-702 & \\
Vertisol (Chromustert), Paget & H 734-758 & $\leq 11,570 \pm 210$ \\
Eutrustox (Krasnozem) Gabbinbar $\quad$ H 719-726 & $\leq 1810 \pm 80$ \\
$\quad$ Eutrustox (Krasnozem), Beechmont (subtropical rainforest) & H 703-711 & $\leq 2020 \pm 70$ \\
\hline
\end{tabular}

\section{Hamburg University, Radiocarbon Dates IV (Scharpenseel, Schiffmann and Becker 1984)}

Soil Samples and Profiles

Tunisia

Fossil gyttja, northwest Degache, Chott el Rharsa

Paleoargid, near Algerian border, underlying fringe of dunes

Paleosol in terrace, Oued Lakarit

Buried Argixeroll, $12 \mathrm{~km}$ from Ksour Essaf

Tirsoid Xerert, Enfida, Station, Amélioration des Parcours

Paleroll, $18 \mathrm{~km}$ from Tadjerouine toward Le Kef

Saem profile fossil horizon $180 \mathrm{~cm}$ deep

Dates of paleosols from perhumid to Saharian climate, $20 \mathrm{~km}$ west of Nefta

North rim of Chott Djerid, $13 \mathrm{~km}$ from Nefta

Humic layer, gravel terrace, rim of Chott

Paleosol, street bridge G P 16, Kebili to Gabes, $62 \mathrm{~km}$ west of Gabes

Polyphasic steppe soil, bank of Oued Ersifa, $25 \mathrm{~km}$ from Matmata

Paleosol in bank of Oued, $5 \mathrm{~km}$ north of Remada, near GP 19

Paleosols in Oued Tatahouine, profile north of Foum Tatahouine

Cut in sediments $300 \mathrm{~m}$ southwest of Matmata-Toujane Street

Sequence of paleosols, south of St. M 201 Gafsa-Moulares, cut in bank of Oued Melah

Polyphasic paleosol, west El Frouch, Djebel Chambi, east of road to Serept

Red relict soil in rock crevices, Sta. Bordj Chambi, Djebel Chambi

Polyphasic paleosol, ca. $800 \mathrm{~m}$ from H 1275-1279

Cut in bank of Oued Bou Hamid, foot of Djebel Semmama

Bank of Oued Bou Hamid, profile 200 m downstream from H 12951304

Transition to terrace at base of previous profile

Organic matter in Oued Bou Hamid terrace, opposite bank to profile H 1305-1312

Duplex Vertisol, northwest Jendouba, north of street to Chamtou, before Satfoura

Polyphasic paleosol, bank of Oued Ogla, $2 \mathrm{~km}$ west of GP 17, Le KefTadjerouine, north bank

Mejerdah alluvium, east Tebourba

Alluvium of Oued Miliane, north of street Pont du Fahs-Smindja, 10 $\mathrm{km}$ from Pont du Fahs

Paleosol in Wadi north of GP 3, Kairouan to Sbeitla

Cut in alluvium of Oued Melize, south of GP 6, Jendouba to Ghardimaou, near bridge

West of GP 1, Tunis-Sfax, $84 \mathrm{~km}$ from Sousse, near crossing to Hammamet Road cut

$\begin{array}{lr}\text { H } 1029 & 2420 \pm 70 \\ \text { H } 1030 & 22,730 \pm 400 \\ \text { H } 1031 & 8050 \pm 100 \\ \text { H } 1032 & 3470 \pm 70 \\ \text { H } 1033 & 4550 \pm 80 \\ \text { H } 1034 & 7960 \pm 110 \\ \text { H } 1035 & 8520 \pm 180 \\ \text { H } 1222-1223 & \leq 10,260 \pm 120 \\ \text { H 1224-1226 } & \leq 4330 \pm 90 \\ \text { H 1227 } & 1950 \pm 60 \\ \text { H 1229 } & 920 \pm 80 \\ \text { H 1233-1236 } & \leq 6420 \pm 130 \\ \text { H 1237, } 1239 & \leq 5200 \pm 160 \\ \text { H 1240,1247 } & \leq 13,490 \pm 220 \\ \text { H 1248-1251 } & \leq 13,530 \pm 370 \\ \text { H 1264-1274 } & \leq 5520 \pm 80 \\ \text { H 1275-1279 } & \leq 7270 \pm 90 \\ \text { H 1283 } & \\ \text { H 1285-1292 } & \leq 4880 \pm 80 \\ \text { H 1295, } 1304 & \leq 3070 \pm 90 \\ \text { H 1305-1311 } & \leq 6860 \pm 100 \\ & \\ \text { H 1311 } 300 \mathrm{~cm} & 14,530 \pm 250) \\ \text { H 1312 } & 9920 \pm 120 \\ \text { H 1313-1318 } & \leq 4670 \pm 90 \\ \text { H 1319-1324 } & \leq 6760 \pm 90 \\ \text { H 1326-1330 } & \leq 5550 \pm 80 \\ \text { H 1334-1340 } & \leq 5850 \pm 90 \\ \text { H 1341-1346 } & \leq 3350 \pm 90 \\ & \\ \text { H 1347-1352 } & \leq 4030 \pm 90 \\ \text { H 1358-1364 } & \leq 11,020 \pm 130 \\ \text { H 1365-1367 } & \leq 780 \pm 80\end{array}$




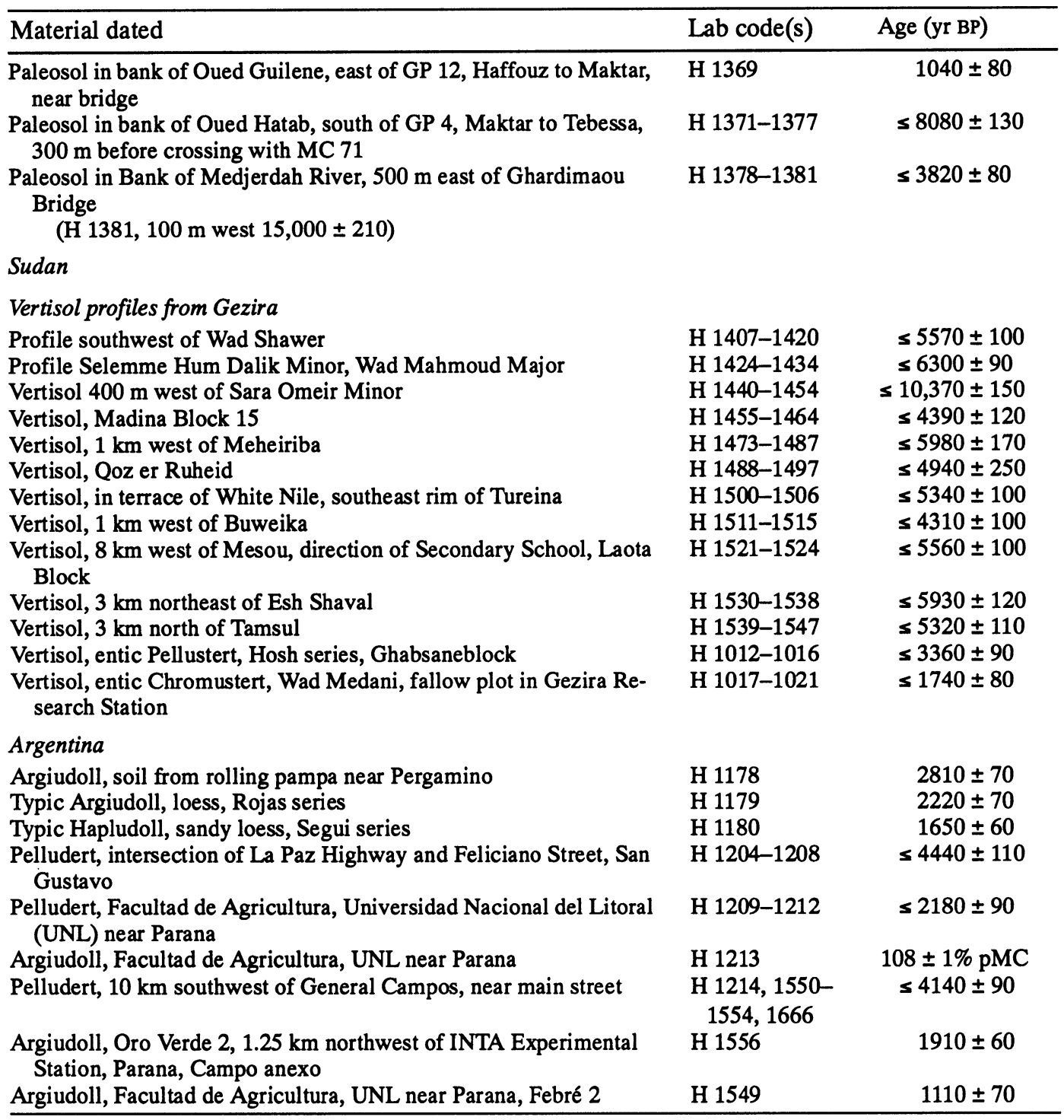

\section{REFERENCES}

Scharpenseel, H. W. and Becker-Heidman, P. 1992 Twenty-five years of radiocarbon dating soils: Paradigm of erring and learning. In Long, A. and $\mathrm{Kra}, \mathrm{R}$. S., eds., Proceedings of the 14th International ${ }^{14} \mathrm{C}$ Conference. Radiocarbon 34(3): 541-549.

Scharpenseel, H. W. and Pietig, F. 1969 Radiokohlenstoff- und Tritium-Datierung von Boden und Wasser durch die Benzolmethode. Geoderma 2: 273-289.

1970 Altersbestimmung mit dem Flüssigkeits Szintillations Spektrometer, Vereinfachte Benzolsynthese, auch aus kleinen CO2-Mengen. Atompraxis 16(3): 1.

1968 University of Bonn natural radiocarbon measurements I. Radiocarbon 10(1): 8-28.
Scharpenseel, H. W. and Pietig, F. 1969 University of Bonn natural radiocarbon measurements II. Radiocarbon 11(1): 3-14.

1970 University of Bonn natural radiocarbon measurements III. Radiocarbon 12(1): 19-39.

1971 University of Bonn natural radiocarbon measurements IV. Radiocarbon 13(2): 189-212.

1973a University of Bonn natural radiocarbon measurements V. Radiocarbon 15(1): 13-41.

1973b University of Bonn natural radiocarbon measurements VI. Radiocarbon 15(2): 252-279.

1974 University of Bonn natural radiocarbon measurements VII. Radiocarbon 16(2): 143-165. 
Scharpenseel, H. W., Pietig, F. and Schiffman, H. 1976 Hamburg University radiocarbon dates I. Radiocarbon 18(3): 268-289.

Scharpenseel, H. W., Pietig, F. and Tamers, M. A. 1968 University of Bonn natural radiocarbon measurements I. Radiocarbon 10(1): 8-28.

Scharpenseel, H. W. and Schiffman, H. 1977 Hamburg University radiocarbon dates II. Radiocarbon 19(2):
170-182.

Scharpenseel, H. W., Schiffman, H. and Becker, P. 1984 Hamburg University radiocarbon dates IV. Radiocarbon 26(3): 367-383.

Scharpenseel, H. W., Schiffman, H. and Hintze, B. 1984 Hamburg University radiocarbon dates III. Radiocarbon 26(2): 196-205.

\section{APPENDIX 1: ANNOTATED BIBLIOGRAPHY}

(Publications based partly to completely on ${ }^{14} \mathrm{C}$ dates on soil and secondary carbonates in soils; arranged in chronological order)

Mückenhausen, E., Scharpenseel, H. W. and Pietig, F. 1968 Zum Alter des Plaggeneschs. Eiszeitalter und Gegenwart 19: 190-196.

- Description of Plaggepts

- Table of three ${ }^{14} \mathrm{C}$-dated Plaggepts, in 10 -cm intervals

- Table of 14 additional Plaggept ${ }^{14} \mathrm{C}$-dating results

Scharpenseel, H. W., Ronzani, C. and Pietig, F. 1968 Comparative age determination on different humic-matter fractions. In Isotopes and Radiation in Soil Organic Matter Studies. Vienna, IAEA: 67-73.

- Fractionation method; table of ${ }^{14} \mathrm{C}$ dating, by $10-\mathrm{cm}$ intervals, of Udoll, Aquoll, Rendoll, Hapludalf, Spodosol soils; table of comparative humus SOM fraction dates of Udalf, two Spodosols, Histosol and Aquoll, indicating highest $\mathrm{C}$ residence time in the Gray humic acid fractions

Scharpenseel, H. W., Tamers, M. A. and Pietig, F. 1968 Altersbestimmung von Böden durch die Radiokohlenstoffdatierungsmethode. Zeitschrift für Pflanzenernährung und Bodenkunde 119(1): 44-52.

- Tables with separate sets of ${ }^{14} \mathrm{C}$ dates: Mollisols of Germany, Hapludalfs, Rendolls and Histosols, Plaggepts, Histosols and Spodosols

Scharpenseel, H. W., Tamers, M. A. and Pietig, F. 1968 Altersbestimmung von Böden durch dei Radiokohlenstoffdatierungsmethode. Zeitschrift für Pflanzenernährung und Bodenkunde 119(1): 34-44.

- Bomb-carbon curve of year-specific field-experimental crop samples and alcohol, distilled from wine of specific origin

- Description of sample preparation and method of benzene synthesis

- Reflection on rejuvenation of soil carbon

- Histogram of all ${ }^{14} \mathrm{C}$ dates known through 1967 , identified by soil types and Quaternary geological origin

Scharpenseel, H. W. and Pietig, F. 1969 Altersbestimmung von Böden durch die Radiokohlenstoffdatierungsmethode. Zeitschrift für Pflanzenernährung und Bodenkunde 122(2): 145-152.

- Table of ${ }^{14} \mathrm{C}$ dates from Hapludalfs, probably formerly Udolls; dating of individual genetic horizons and subhorizons; the highest ${ }^{14} \mathrm{C}$ ages always occur in argillic horizons

- Table with ${ }^{14} \mathrm{C}$ dates of paleosols (Mollisols), some $>10,000 \mathrm{BP}$

Scharpenseel, H. W. 1972 Natural radiocarbon measurement of soil and organic matter fractions and on soil profiles of different pedogenesis. Proceedings of the 8th International Conference on Radiocarbon Dating. Wellington, New Zealand, Royal Society: 384-393.

- Table of ${ }^{14} \mathrm{C}$ dates on SOM fractions from Histosol, Spodosols, Aquoll, Udoll and a Paleudoll

- Table of ${ }^{14} \mathrm{C}$ dates of different particle fractions of silt and clay, compared with charcoal samples; highest age among particle fractions in fine silt and coarse clay

- Diagrams with date-points: regression line and correlation equation for available dates of Spodosols, Alfisols, Udolls, Plaggepts and Vertisols

- Age vs. depth curves of 39 Vertisols in diagram, split into six fields of different countries of origin 
Scharpenseel, H. W. 1972 Messung der natürlichen C-14 Konzentration in der organischer Substanz von rezenten Böden. Eien Zwischenbilanz. Zeitschrift für Pflanzenernährung und Bodenkunde 133(3): 241-263.

- Balance of work: Reference collection, 12 figures and 3 tables, summarizing all our ${ }^{14} \mathrm{C}$-dating of soil samples, profile scans, SOM-fractions and integral regression/correlation curves

Scharpenseel, H. W. 1974 Zur Berechtigung der Interpretation natürlicher Radiokohlenstoffmessungen an organischem Kohlenstoff $\mathrm{CaCO}_{3}$-reicher Böden. Mitteilungen der Deutschen Bodenkundlichen Gesellschaft 18: 198-201.

- Seven Udoll, Vertisol and Hapludlf profiles from Czechoslovakia, Bulgaria, Italy and Germany; ${ }^{14} \mathrm{C}$ dating and $\delta^{13} \mathrm{C}$ measurement in all genetic horizons

Scharpenseel, H. W. 1975 Relative age sequence of fractions of soil organic matter. Application of Nuclear Methods in Biology and Agriculture. ENSA (European Society of Nuclear Methods in Agriculture) 5: 10-17.

- Table of SOM fraction dating, including Sephadex gel fractionation

- Setup for preparative gel fractionation as a graph

Scharpenseel, H. W. 1975 Relativalter und Sukzession von Fraktionen der organischen Bodensubstanz. Mitteilungen der Deutschen Bodenkundlichen Gesellschaft 22: 453-466.

- Soil fraction ${ }^{14} \mathrm{C}$ dating; all available fractionation methods are compared. Dimethylsulfoxide extract as well as $6 \mathrm{~N} \mathrm{HCl}$ hydrolysis residue seem to produce the highest ${ }^{14} \mathrm{C}$ ages

Scharpenseel, H. W. and Schiffmann, H. 1977 Radiocarbon dating of soils, a review. Zeitschrift für Pflanzenernährung und Bodenkunde 140: 159-174.

- Review of the state of the art

Scharpenseel, H. W. 1977 The search for biologically inert and lithogenic carbon in recent soil organic matter. Soil Organic Matter Studies. Volume 2. Vienna, IAEA: 193-200.

- All fractionation methods including gel fractions and repetitive $6 \mathrm{~N} \mathrm{HCl}$-hydrolysis dating results and comparison. Repetitive acid hydrolysis seems to be the best method, but laborious sample preparation methods prohibit routine use.

Blackburn, G., Sleeman, J. R. and Scharpenseel, H. W. 1979 Radiocarbon measurements and soil micromorphology as guides in the formation of Gilgai at Kaniva, Victoria. Australian Journal of Soil Research 17: 115.

- Four Vertisol profiles, sampled in 10-cm intervals; one mound and one depression profile each from Lillimur and Miram were ${ }^{14} \mathrm{C}$-dated. Mound samples are superior in carbon residence time to depression samples.

Meyer-Spasche, H. and Scharpenseel, H. W. 1980 Die Grundbelastung von Böden mit ${ }^{14} \mathrm{C}$ und dessen Transfer zum Menschen. Zeitschrift für Pflanzenernährung und Bodenkunde 143(5): 537-545.

- Bomb-carbon curve up to 1980 , mainly from wine and grass

- Bomb-carbon transfer $\mathrm{pCi} / \mathrm{gC}$ in grass, milk and human hair

- Natural ${ }^{14} \mathrm{C}$ activity by layer in a pine forest, pasture and cropland, also as ${ }^{14} \mathrm{C}$ activity $\mathrm{pCi} / \mathrm{gC}$

Stephan, S., Berrier, J., De Petre, A. A., Jeanson, C., Kooistra, M. J., Scharpenseel, H. W. and Schiffman, H. 1983 Characterization of in situ organic matter constituents in Vertisols from Argentina, using submicroscopic and cytochemical methods. First report. Geoderma 30: 21-34.

- Carbon dating of Vertisol profiles as part of very detailed soil investigations

Schleser, G. H., Bertram, H. G. and Scharpenseel, H. W. 1983 Aussagen über Bildungsprozesse tunesischer Kalkkrusten mittles ${ }^{13} \mathrm{C} /{ }^{12} \mathrm{C}$-Isotopenanalysen. Mitteilungen der Deutschen Bodenkundlichen Gesellschaft 38: 573-578.

- Checking the ad ascensum and ad descensum hypothesis of calcrete formation by changes in ${ }^{13} \mathrm{C}$ concentration throughout a profile 
Scharpenseel, H. W. and Neue, H. U. 1984 Use of isotopes in studying the dynamics of organic matter in soils. Proceedings on the Conference of Humus in Rice Soils. IRRI, Philippines: 273-310.

- Comprising overview of tracer and low-level $\left({ }^{14} \mathrm{C}\right.$ dating) studies in relation to organic-matter turnover and SOM-C residence time in terrestrial and submerged soils

Scharpenseel, H. W. and Schiffman, H. 1985 Natürliche Radiokohlenstoffmessungen als Beitrag zur Definition rezent- oder paläoklimatischer Leithorizonte in Tunesien. Zeitschrift für Pflanzenernährung und Bodenkunde 148(2): 113-130.

- Large list of ${ }^{14} \mathrm{C}$-dated paleosols and geomorphological indicator horizons; histogram of all soil and groundwater ${ }^{14} \mathrm{C}$ dates from Tunisia

- Reflection on carbon residence time in different climate zones and on climate fluctuations favoring formation of soils and phreatic groundwater deposits

Becker-Heidmann, P. and Scharpenseel, H. W. 1986. Thin layer $\delta^{13} \mathrm{C}$ and $\mathrm{D}^{14} \mathrm{C}$ monitoring of "Lessive" soil profiles. In Stuiver, M. and Kra, R., eds., Proceedings of the 12 th International ${ }^{14} \mathrm{C}$ Conference. Radiocarbon 28(2A): 383-390.

- Thin layer scanning of ${ }^{14} \mathrm{C}$ and $\delta^{13} \mathrm{C}$ in two well-analyzed forest soils (Hapludalfs) for tracing of carbon dynamics

Mtimet, A., Freytag, J. and Scharpenseel, H. W. 1986 Investigations isotopiques $\left(\delta^{13} \mathrm{C}\right.$ and $\left.\delta^{18} \mathrm{O}\right)$ des croûtes calcaires et encroûtements des Matmata et de la Plaine de Jeffara en Tunisie. Extended abstract accompanying poster in Proceedings of the 13th International Soils Conference, Hamburg, Germany: 1217.

- $\delta^{13} \mathrm{C}$ and $\delta^{18} \mathrm{O}$ scan of calcretes in Tunisia exploring the formation mechanism

- A few accompanying ${ }^{14} \mathrm{C}$ dates were either obviously rejuvenated or in a deeper position (very old) but not old enough to indicate formation (due to atmospheric C-component)

Scharpenseel, H. W., Freytag., J. and Becker-Heidmann, P. 1986 C-14 Alterbestimmung und $\delta^{13}$ C-Messungen an Vertisolen unter besonderer Berücksichtigung der Gezieraböden des Sudan. Zeitschrift für Planzenernährung und Bodenkunde 149: 277-289.

- Assessment of regression curves for $378{ }^{14} \mathrm{C}$ dates of Vertisols from 10 countries

- $\delta^{13} \mathrm{C}$ profile curves indicating change of $\mathrm{C}_{3}$ (cotton, underlying Nile alluvium) and $\mathrm{C}_{4}$ vegetation (durra)

- Carbon migration into clayey smectitic soils

Scharpenseel, H. W., Tsutsuki, K., Becker-Heidmann, P. and Freytag, J. 1986 Untersuchung zur Kohlenstoffdynamik und Bioturbation von Mollisolen. Zeitschrift für Pflazenernährung und Bodenkunde 149: 582-597.

- Mollisol (from eight countries) ages vs. depth curves; regression and correlation equations; age/profiletop-age vs. depth curves

- Comparison of ${ }^{14} \mathrm{C}$ dates in different texture fractions of soils

- ${ }^{14} \mathrm{C}$ dates of earthworm- $\mathrm{C}$ from different soil depth layers indicating that body-C of earthworms (Eisenia) is fully recent (bomb-C), foreign to the 4500 -yr-old $\mathrm{C}$ of soil humus

Becker-Heidman, P., Liu Liang-wu, Scharpenseel, H. W. 1988 Radiocarbon dating of organic matter fractions of a Chinese Mollisol. Zeitschrift für Pflazenernährung und Bodenkunde 151: 37-39.

- Relations between SOM fraction dates and soil depth.

Scharpenseel, H. W. and Becker-Heidman, P. 1989 Shifts in ${ }^{14} \mathrm{C}$ patterns of soil profiles due to bomb carbon, including effects of morphogenetic and turbation processes. Radiocarbon 31(3): 627-636.

- Bomb carbon curve to 1988

- Admixture of recent $\mathrm{C} v s$. shift from mean residence time to apparent mean residence time

- Organic matter decomposition

- Bomb-carbon penetration into depth of profiles of different soil types, humidity

- Alfisol, Inceptisol, Mollisol, Spodosol, Vertisol ages vs. depth regression curves, regression and correlation factor

- $\delta^{13} \mathrm{C}$ curve in Vertisol with $\mathrm{C}_{3}$ and $\mathrm{C}_{4}$ plant alternation

- ${ }^{14} \mathrm{C}$ depth curve of earthworms in Mollisol layers 
Scharpenseel, H. W., Becker-Heidman, P., Neue, H. U. and Tsutsuki, K. 1989 Bomb-carbon, ${ }^{14} \mathrm{C}$-dating and ${ }^{13} \mathrm{C}$ measurements as tracers of organic matter dynamics as well as of morphogenetic and turbation processes. The Science of the Total Environment 81/82: 99-110.

- Bomb carbon in soil profiles

- Thin-layer scan by ${ }^{14} \mathrm{C}$ dating in terrestrial and submerged rice soil

- Regression curves for profile dates of different soil types

Becker-Heidman, P. and Scharpenseel, H. W. 1989 Carbon isotope dynamics in some tropical soils. In Long, A., Kra, R. S. and Srdoc, D., eds, Proceedings of the 13 th International ${ }^{14} \mathrm{C}$ Conference. Radiocarbon 31(3): 672-679.

- Thin-layer scan of ${ }^{14} \mathrm{C}$ measurements in different profiles of tropical soils.

Neue, H. U., Becker-Heidman, P. and Scharpenseel, H. W. 1990 Organic matter dynaics, soil properties and cultural practices in ricelands and their relationship to methan production. In Bouwman, A. F., ed., Soils and the Greenhouse Effect. New York, Wiley: 456-466.

- Thin-layer scan of rice fields by ${ }^{14} \mathrm{C}$ dating and especially $\delta^{13} \mathrm{C}$, indicating the zone of methanogenesis in submerged soil profiles

Scharpenseel, H. W., Neue, H. U. and Singer, St. 1992 Biotransformations in different climate belts; source-sink relationships. In Kubat, K., ed., Humus, Its Structure and Role in Agriculture and Environment. Elsevier: 91105.

- Within context of biomass turnover, some minor supplements to low-level studies

Scharpenseel, H. W. and Becker-Heidman, P. 1993 The dilemma of conflicting interests between $\mathrm{CO}_{2}$ 's and $\mathrm{CH}_{4}$ 's IR trapping capacity and role, in case of $\mathrm{CO}_{2}$ as limiting factor for plant growth. Proc. Global Warming - A Call for International Coordination. SUPCON International. World Resource Review 4(2): 242-258.

- Some thin-layer scanned soil profiles for carbon residence time

Scharpenseel, H. W. and Becker-Heidman, P. 1992 Twenty-five years of radiocarbon dating soils: Paradigm of erring and learning. In Long, A. and Kra, R. S., eds., Proceedings of the 14 th International ${ }^{14} \mathrm{C}$ Conference. Radiocarbon 34(3): 541-549.

- Implications of ${ }^{14} \mathrm{C}$ dating of soils: rejuvenation; compartmental exchange; carbon elluviation and illuviation; transfer by turbation

Scharpenseel, H. W. 1994 Sustainable land use in the light of resilience/elasticity to soil organic matter fluctuations. In Greenland, D. and Szabolcs, I, eds., Soil Resilience and Sustainable Land Use. Proceedings of the Symposium, Budapest 28 September to 2 October 1992. Wallingford, CAB International: 249-264.

- ${ }^{14} \mathrm{C}$ dates due to soil profile scan indicating carbon dynamics

- ${ }^{14} \mathrm{C}$ dates due to profile scan reflecting preferential link of $\mathrm{C}$ to clay minerals by clay-organic complexation 
APPENDIX 2: PREVIOUSLY UNPUBLISHED SOIL DATES

\begin{tabular}{|c|c|c|}
\hline Lab code & Site & Age (yr BP) \\
\hline \multicolumn{3}{|c|}{ Four samples from Mosbruch, base of Histosol } \\
\hline HAM 1557 & Mosbruch 1 & $11,760 \pm 435$ \\
\hline HAM 1558 & Mosbruch 2 & $11,580 \pm 500$ \\
\hline HAM 1559 & Mosbruch 3 & $12,200 \pm 500$ \\
\hline HAM 1560 & Mosbruch 4 & $11,980 \pm 435$ \\
\hline HAM 1566 & $\begin{array}{l}\text { Fossil argillic horizon, Tunisia, } 50-60 \mathrm{~cm} \text {, sand dunes } 4 \mathrm{~km} \text { east } \\
\text { of Algerian border, south Tozeur }\end{array}$ & $430 \pm 200$ \\
\hline HAM 1568 & Sample of Vertisol, Argentina, San Gustavo, $130-150 \mathrm{~cm}$ & $5600 \pm 100$ \\
\hline \multicolumn{3}{|c|}{ Channel cast, Waldsee } \\
\hline HAM 1570 & Waldsee $1,99-104 \mathrm{~cm}$, lightly decomposed peat & $3440 \pm 80$ \\
\hline HAM 1571 & Waldsee $2,199-204 \mathrm{~cm}$, strongly decomposing peat & $4200 \pm 80$ \\
\hline HAM 1573 & Waldsee 4, $209-219 \mathrm{~cm}$, fossil stem material, Alnus & $4630 \pm 80$ \\
\hline HAM 1572 & Waldsee 3, 295-300 cm fine detritral gyttja and plant remains & $20,600 \pm 350$ \\
\hline \multicolumn{3}{|c|}{ Relics found in soil profile } \\
\hline HAM 1574 & Knodl 1, floating wood pieces & $9220 \pm 100$ \\
\hline HAM 1575 & Knodl 2, floating wood pieces & $25,500 \pm 900$ \\
\hline HAM 1576 & Grapel, pine wood at base of low moor & $8700 \pm 160$ \\
\hline \multicolumn{3}{|c|}{$\begin{array}{l}\text { Peat and gyttja samples of palynologically tested profile, Moor (peat) Plidutscha, Canton } \\
\text { Graubünden, Switzerland }\end{array}$} \\
\hline HAM 1587 & VM $7.3 \mathrm{~A}$, peat, $24-35 \mathrm{~cm}$ & $2710 \pm 85$ \\
\hline HAM 1588 & VM $7.2 \mathrm{~A}$, peat, $50-60 \mathrm{~cm}$ & $910 \pm 140$ \\
\hline HAM 1589 & VM $7.1 \mathrm{~A}$, peat, $77-87 \mathrm{~cm}$ & $620 \pm 150$ \\
\hline HAM 1590 & VM $3.1 \mathrm{~B}$, peat with gyttja, $181-192 \mathrm{~cm}$ & $2810 \pm 120$ \\
\hline HAM 1591 & VM $3.1 \mathrm{~A}$, gyttja with peat, $195-207 \mathrm{~cm}$ & $3380 \pm 120$ \\
\hline HAM 1592 & VM 4.2, gyttja, $229-242 \mathrm{~cm}$ & $3820 \pm 150$ \\
\hline HAM 1593 & VM 4.1, gyttja, $256-270 \mathrm{~cm}$ & $4990 \pm 140$ \\
\hline HAM 1594 & VM 5.3, gyttja, $280-292 \mathrm{~cm}$ & $5130 \pm 145$ \\
\hline HAM 1595 & VM $6.2 \mathrm{~A}$, clayey gyttja, $382-390 \mathrm{~cm}$ & $9050 \pm 250$ \\
\hline HAM 1597 & Sediment from Hamburg harbor basin & $1730 \pm 80$ \\
\hline
\end{tabular}

